\title{
L'Assurance et l'Intégration Monétaire Européenne
}

\author{
par Fabio Padoa *
}

Le monde de l'assurance porte un grand intérêt à l'intégration économique et monétaire européenne. Je vais citer trois aspects sous lesquels cet intérêt est manifeste.

D'abord au titre de l'objet même de l'assurance, de sa raison d'être. On peut dire que l'assurance c'est le refus de la fatalité. C'est par définition un pari sur l'avenir. L'assurance se conçoit seulement dans un climat de confiance et de stabilité ; elle exprime de la part du preneur d'assurance la volonté de s'affirmer, de durer, en dépit des circonstances adverses. L'assurance, quelqu'un l'a dit, c'est le contrat de l'espoir, et c'est aussi le contrat de l'effort personnel contre la menace de revers ou d'infortunes possibles. Donc tout ce qui va dans le sens de la stabilité politique et monétaire va dans le sens souhaité par le preneur d'assurance, en renforçant sa confiance constructive dans l'avenir. La stabilité des institutions favorise donc l'esprit de prévoyance, et l'assurance qui l'incarne.

En deuxième lieu, l'assurance est intéressée à la stabilité du point de vue de ses entreprises elles-mêmes. Tout le monde sait qu'il est caractéristique pour notre activité d'opérer en régime d'inversion du cycle entre les coûts et les rentrées, puisque nous ne connaissons le prix véritable du risque qu'a posteriori, quand tous les sinistres ont été liquidés. Or, l'assurance est un mécanisme lent : il se produit un décalage sensible entre le moment où on conçoit un tarif et le moment où on l'applique, entre le moment de la souscription d'un contrat et le moment de la prestation, entre le moment où un sinistre survient et le moment où il est liquidé. $\mathrm{Si}$ dans cet intervalle ou dans ces différents intervalles, le mètre monétaire change de longueur, évidemment tous les calculs sont faussés. Il y a lieu d'ajouter que le niveau de nos primes, même lorsqu'il est indexé au coût de la vie, est nécessairement en retard sur le coût des prestations. Pour la bonne raison qu'à peu près $90 \%$ des débours d'une société d'assurance sont en fonction des salaires, soit des salaires qu'elle paye à ses employés, soit des salaires des ateliers de réparation, soit enfin des salaires des tiers lésé. Or, c'est un fait bien connu que l'indice des salaires grimpe toujours plus vite que l'indice du coût de la vie. Et il est déjà malaisé pour l'assureur de faire comprendre que ses primes doivent suivre le trend du coût de la vie. Donc dans un régime d'inflation le prix de l'assurance est perpétuellement en retard.

D'autre part, la prudente répartition de nos actifs sur laquelle repose la stabilité des bilans est bouleversée par les fluctuations des valeurs exprimées en termes monétaires. Nous croyons avoir investi $25 \%$ dans les immeubles et, que sais-je, $10 \%$ dans les actions, et voici que ces proportions changent et on se trouve devant un tableau

* Président de l'« Association de Genève ». 
totalement déformé. La réglementation communautaire sur la marge de solvabilité selon laquelle les moyens propres de l'entreprise d'assurance doivent croître parallèlement à son chiffre d'affaires, aboutit, dans une période d'inflation, à un véritable paradoxe fiscal : pour la part de croissance qui du fait de l'inflation est plus apparente que réelle, le prélèvement d'impôts sur les bénéfices, souvent apparents eux aussi, finit par affaiblir l'entreprise au lieu d'en accroître la sécurité. C'est un problème sérieux, qu'il faudra bien affronter un jour.

Enfin, en période d'inflation, nos bilans risquent d'être faux. Ils le sont même nécessairement dès que, faisant l'addition des poires et des pommes, nous mettons ensemble des évaluations faites en des époques différentes : ils ne sont donc plus ce qu'ils devraient être, c'est-à-dire un instantané de la situation réelle à un moment donné. Et ce qui est plus grave c'est que souvent, nous ne nous en apercevons même pas. Nous croyons à nos chiffres alors qu'ils ne représentent que des apparences et nous ne nous apercevons qu'en retard que nos progrès sont factices et que l'inflation nous a appauvris. Là encore, une conduite saine de l'entreprise d'assurance n'est concevable que dans un régime de stabilité monétaire.

Troisièmement, le rôle de l'assurance dans la société. L'une de nos tâches est de donner une réponse concrète au problème de la sécurité, de rendre l'insécurité tolérable, cette insécurité foncière, caractéristique de notre époque.

Or, ce rôle est rendu plus difficile voire impossible par une situation d'instabilité généralisée. J'ai entendu dire dans une réunion récente que finalement les calamités naturelles, les tremblements de terre, les grands incendies, les naufrages que nous assurons, représentent un risque de faible ampleur en comparaison du risque catastrophique de fluctuation des valeurs inscrites dans nos bilans. Nous devons faire, par définition, par vocation, des investissements à long terme, puisque nous sommes non seulement des récolteurs d'épargne individuelle, mais également des créateurs d'épargne collective. La technique même de l'assurance nous oblige à mettre de côté certaines sommes que l'individu ne mettrait pas de côté dans le même but. Ces sommes sont mises sous forme de réserves techniques à la disposition de la collectivité pour des investissements à long terme. Or, si ces investissements se révèlent en partie illusoires, notre apport financier perd une partie de son efficacité sociale.

Enfin, sur un plan politique, là aussi il faut dire que la véritable dimension de l'assurance est plus que nationale. Déjà aujourd'hui, l'assurance libre échappe au cloisonnement propre à l'assurance sociale qui, fatalement, est toujours enfermée dans un cadre administratif national : alors que nous, nous ne pouvons exercer notre métier que si nous opérons, ne fût-ce que par le biais de la réassurance, dans un cadre international. Or, pouvoir sous cette optique se soustraire aux aléas des changes, ce serait pouvoir mieux exercer notre métier, ce serait pouvoir mieux faire face aux dimensions des risques qui augmentent constamment, ce serait pouvoir mieux répondre à la demande croissante de capacité des marchés, pouvoir donner une meilleure contribution à la protection contre les nouveaux risques. Voici aussi pourquoi, pour notre métier, nous souhaitons la stabilité monétaire.

Mais notre métier encore, c'est d'encourager l'épargne individuelle à travers l'assurance. Alors quand nous entendons le Professeur Triffin nous parler de l'écu comme d'une monnaie qui pourra un jour - et peut-être même un jour pas trop 
éloigné - servir non seulement aux transactions entre les banques centrales mais parvenir au niveau des privés, notre imagination d'assureurs pense tout de suite à des polices d'assurance où l'épargne se ferait en écus : c'est-à-dire dans une monnaie dont la stabilité serait bien supérieure à celle de la plupart des monnaies nationales actuelles. Ce seul fait donnerait à l'épargne par la voie de l'assurance une impulsion considérable.

L'intégration monétaire européenne nous intéresse aussi à un autre chef. Il me paraît évident que, dans un cadre élargi tel que celui qui se dessine avec la Communauté, une rationalisation des marchés pourrait se vérifier plus aisément au niveau des entreprises. Nous n'avons pas encore cette société européenne que certains demandent à grand cris, mais qui rencontre aussi, nous le savons, de fortes oppositions. Eh bien, si l'obstacle monétaire était éliminé ou dans la mesure où il serait éliminé, il est évident que certaines difficultés objectives qui subsistent aujourd'hui pour les concentrations d'entreprises - par exemple pour des concentrations électives entre petites et moyennes entreprises ayant une même vocation - pourraient se faire plus facilement au-delà des frontières, et que par ce biais on pourrait arriver à une rationalisation de nos activités.

Monsieur Jenkins, quand il a parlé à Florence, je crois fin 1977 , nous a rappelé que dans la nature l'évolution n'est pas continue, que c'est une évolution par sauts ; et c'est justement un saut de qualité que nous attendons d'événements comme l'élection du Parlement européen au suffrage universel, et la mise en place d'un nouveau système monétaire européen.

Je crois pouvoir dire au nom de tous mes collègues assureurs que c'est avec espoir et conviction que nous envisageons les possibilités futures d'un tel saut de qualité. 